57

\section{HEALTH-RELATED QUALITY OF LIFE IN}

C Cunningham $^{1}$, D Drotar ${ }^{1}$, T. M. Palermo ${ }^{2}$, K. McGowan ${ }^{3}$, Robert Arendt ${ }^{4},{ }^{1}$ Rainbow Babies \& Children s Hospital and Department of Pediatrics, Case School of Medicine, ${ }^{2}$ Department of Anesthesiology and Peri-Operative Medicine, Oregon Health \& Sciences

pology, St. Mary's College of Maryland and The Buckeye Ranch
This study examined the health-related quality of life (HRQOL) of children with Inflammatory Bowel Disease (IBD) compared with physically healthy children and the impact of IBD and steroidal side-effect on children's HRQOL. The HRQOL of 49 children and adolescents (ages 10 to 18 years) with IBD and their parents who completed the Child Health Questionnaire was compared to that of a sample of physically healthy children $(\mathrm{N}=49)$. Caregivers of children with IBD reported that their children' physical and psychological health were more limited and that they experienced more emotional worry and greater impact on their personal time compared to caregivers reporting on healthy same-age children. Children and adolescents reported worse HRQOL than physically healthy children in only one domain: general health. Children and adolescents with more IBD-related and steroidal symptoms had less adequate $\mathrm{HRQOL}$ as reported by their parents. Limitations in $\mathrm{HRQOL}$ were most pronounced in children who experienced more frequent $\mathrm{BBD}$-related symptoms together with symptoms of steroidal side effects. Key Words: inflammatory bowel disease, quality of life, medication side effects, children

\section{8}

TOPICAL/ORAL NYSTATIN FOR PREVENTION OF INVASIVE FUNGAL DISEASE IN EXTREMELY LOW BIRTH WEIGHT (ELBW) INFANTS.

HB Srinivasan, O Ajayi, G Srinivasan, Sinai Children's Hospital, Chicago, IL.

BACKGROUND: Invasive fungal infection is associated with significant morbidity and mortality in preterm infants. Studies have suggested that colonization with Candida species is an important risk factor for subsequent invasive fungal disease. Prophylactic treatment with fluconazole has shown to decrease the incidence of fungal sepsis in ELBW infants, but risk of toxicity and emergence of resistance are important concerns. The incidence of invasive fungal disease in ELBW infants reported in literature range from 3.8-12\%. OBJECTIVE: To document the incidence of invasive fungal disease in ELBW infants in a neonatal intensive care unit (NICU) that routinely uses prophylactic topical and oral nystatin application in ELBW infants. DESIGN/METHODS: Data was collected by retrospective review of medical records of all ELBW infants admitted to the NICU of Sinai Children's Hospital over a 8 yea period from 100 to 2004. All ELBW infants admitted received 0.5 ml of nystatin oral suspension $(100,000$ units/ml) orally twice daily and nystatin topical ointment to the perineal and periumbilical region twice daily for the first four weeks of life. No routine surveillance cultures were obtained. Infant eriod, 354 ELBW infals wich perod, 354 ELBWUSIONS: The aniled the NICU and 12 ind disease. CONCLUSIONS: The incidence on Wasive fungal disease in ELBW sto in to topical/oral nystatin. Further randomized controlled clinical trials need to be conducted to compare the

\section{9}

POTENTIAL UTILITY OF BRAIN NATRIURETIC PEPTIDE IN THE EVALUATION OF THE DYSPNEIC CHILD WITH CONGENITAL HEART DISEASE.

R. McCulloh, S. Mato, University Hospital and Clinics, Columbia, MO.

BACKGROUND: The use of brain natriuretic peptide (BNP) in the evaluation of the dyspneic pediatric patient is increasingly supported for infants and newborns with congenital heart disease child or a loscent with congenital heart disease. OBJECTIVE. To report the utility of BNP in differentiating heart failure from other causes of dyspnea in the child with congenital heart disease well as providing insight into the effectiveness of heart failure therapy. DESIGN/METHODS: Case report

RESULTS: A 13 year old male with Down Syndrome and previous AV canal repair and mitral valve replacement with porcine xenograft in 1998 was admitted to our center for evaluation of increasing fatigue, dyspnea, and orthopnea. These symptoms gradually developed over the past 7 weeks and were associated with a $4 \mathrm{~kg}$ weight gain over the past month. The patient had also had a previous episode of viral myocarditis and had undergone treatment with enalapril, spironolactone, and digoxin for severa months in 2004 , but was currently only on iron, digoxin, and warfarin.

Upon admission the patient was shown to have marked dyspnea, hypoxemia requiring 5 liters per minute oxygen by nasal canula, some trace anterior tibial edema, and prominent JVD. Echocardiogram showed increased left atrial pressures and mitral valve stenosis. BNP was drawn at admission and was found to be $5486.91 \mathrm{pg} / \mathrm{ml}$. He was given $40 \mathrm{mg}$ furosemide in addition to his home medications and was started on a low-sodium diet. During the night of hospital day 1 the patient's dyspnea improved and his oxygen requirement resolved. On hostpital day 2 the patient was started on enalapril $2.5 \mathrm{mg}$ daily and spironolactone $12.5 \mathrm{mg}$ daily, and digoxin was increased to $0.125 \mathrm{mg}$ twice daily. By hospital day 5 the patient's BNP had decreased to $4007.77 \mathrm{pg} / \mathrm{ml}$, his appetite was back to baseline, and he was ambulating and sleeping flat without difficulty. The patient was transferred to another facility for replacement of his deteriorating mitral valve. CONCLUSIONS: BNP shows promise in differentiating heart failure from non-cardiac causes of dyspnea in the child with congenital heart disease and prior surgical intervention.
Additionally, BNP level decrease correlated with clinical improvement in our patient. This may prove insightful in assessing the efficacy of heart failure therapy in this specialized population.

First Author is a House Officer
60

PHARMACOLOGICAL MANAGEMENT OF OBESITY IN CERTAIN SYNDROMES: A CASE REPORT.

C Urbanowicz, F Ziai, Advocate Hope Children's Hospital, Oak Lawn, IL. sleep apnea. We have previously reported successful treatment of hyperinsulinemic obesity syndrome in otherwise normal children and adolescents with amphetamine salts. Our objective was to investigate the patients with Down and Prader Willi Syndrome who responded very well to pharmacological treatment, hort term

Patient Number 1: B.C. is a 9 year old female who is a known case of trisomy 21 under treatment for autoimmune hypothyroidism, which was well maintained with thyroxine treatment. She was at the $10^{\text {th }}$ percentile for height at the age of 10 years old. She weighed 90 pounds, which was 30 pounds over her ideal body weight and placed her at the $90^{\text {th }}$ percentile for weight. She began treatment with amphetamine mixed compounds, Adderall XR. During the ens

Patient Number 2: G.M. is a 12 year old female child with a known case of Prader Willi Syndrome with moderate mental retardation. She presented at the age of 10 years with her height at the $3^{\text {rd }}$ percentile an

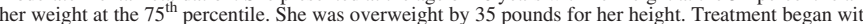
amphetamine mixed compounds, Adderall XR. She too responded favorably by growing along the $3^{\text {th }}$ percentile while her BMI progressively decreased from 26 to $21.4,19.2$ and 18.8 within a 13 month
period. Both patients tolerated this treatment very well. There were no adverse side effects and anecdotally their parents noted improvements in their mental function.

\section{1}

MENSES SUPPRESSION IN AN ADOLESCENT WITH VAGINAL AGENESIS. S Rahhal, E Walvoord, Indiana University School of Medicine, Indianapolis, IN

Background: Mayer-Rokitansky-Kuster-Hauser Anomaly (MRKHA) exists within a spectrum rang ing from the complete absence of all müllerian structures to the presence of a normal uterus but vagina agenesis. Patients with this disorder have normal ovarian function and normal pubertal development, bu primary amenorrhea. In girls with a uterus and an anatomic outflow obstruction, endometrial build up can lead to significantly increased pressure in the genital tract with retrograde flow of menstrual blood resulting in an increased risk of endonetriosis and abdominal pain. The optimal clinical management of pres pon is unk present wan. Current treatent options for dienses suppression in parient we a previon revealed hematosalpinx and absence of a vagina. The right fallopian tube was severely distended an vanis were identified; GnRHa supprin and enct progesterone , GnRHer suppression, depo moxy progestere purpose of suppressing menses in a healthy adolescent girl were found. Due to theoretical concern regarding adverse effects on bone mineral density with the other two choices, the decision was made to initiate treatment with the estrogen-progesterone patch. However after several months on treatment, she was 12 hours late in changing her patch and presented to the emergency room with abdominal pain
Pelvic ultrasound showed uterine and pelvic blood. She was then changed to a $30 \mu \mathrm{g}$ estrogen with Pelvic ultrasound showed uterine and pelvic blood. She was then changed to a $30 \mu \mathrm{g}$ estrogen with of bleeding have been noted over the past 8 months. Conclusions: Complete suppression of menses in an otherwise normal child is an infrequent goal of clinical management. This case emphasizes the difficulty of choosing the ideal pharmacologic strategy and highlights the lack of long term therapeutic outcome data in similar patients. Prospective controlled studies are needed to investigate safety and efficacy of different therapeutic modalities for menses suppression in those who choose to delay surgical correction of a non-patent outflow tract. 